\title{
Spatial convolution of a stress field analyzed by X-ray diffraction
}

\author{
C. Kahloun ${ }^{*, a}$, R. Badji ${ }^{2, b}$, S. Queyreau ${ }^{1, c}$, P. Franciosi ${ }^{1, c}$, B. Bacroix ${ }^{1, c}$ \\ ${ }^{1}$ LSPM-CNRS, UPR 3407, Université Paris 13, 93430 Villetaneuse, France \\ 2 Welding and NDT Research centre (CSC) B.P 64, Cheraga Alger \\ a-charlie.kahloun@Ispm.cnrs.fr ${ }^{b}$ riadbadji@gmail.com
}

c-sylvain.queyreau@.cnrs.Ispm.fr, patrick.franciosi@Ispm.cnrs.fr,brigitte.bacroix@lspm.cnrs.fr

Keywords: XRDstress measurement-spatial convolution-stress gradient-inverse problem

\begin{abstract}
X-ray stress analysis suffers from homogeneity limitations of the stress field in the analyzed volume. When this homogeneity is not fulfilled, it is possible to reduce the irradiated volume down to stress homogeneity achievement. New limitation however occurs : the diffracting sites become too few for stress homogenization. We show that the diffractometry analysis corresponds to a spatially convoluted stress field. The inverse convolution problem is posed. An example of regularization method is given.
\end{abstract}

\section{Introduction:}

X-Ray Diffraction (XRD) is a powerfull tool to determine residual stresses existing in specimens. However, conventional procedures assume stresses to be homogeneous over the irradiated zone [1], which may not be the case. When residual stresses vary over a distance smaller that the irradiated zone, the stress measures obtained from XRD correspond to the convolution of the local stress over the analysed area. It is expected that the incidence angle may also affect the measures as the incidence angle modifies the irradiated zone geometry. Deconvolution preocedures can be usefull to obtain the local stress field from the convoluted stress measures. However, deconvolution is a illposed inverse problem and noise in the experimental data can lead to instability in the solution. The question of the spatial convolution of stress fields analyzed by XRD has rarely been addressed in the literature $[2,3]$. In this paper, we assume that the XRD stress measurements result from the spatial convolution of the local stress field that varies over a domain smaller than the irradiated zone. We first characterize the convolution window using the response of the diffractometer to a stress step function, and we suggest an approximate expression of the impulse response of the diffractometer. The characteristics of this impulse response will then be studied according to the selection of the incidence angles. Finally, an example of a deconvolution procedure of the experimental stress field is provided.

\section{Experimental procedure}

X-Ray equipment distributed by Siemens [4] is used in the present work. For a given incidence angle $\Psi$, the irradiated zone exhibits a shape of a disc whose diameter can be varied using an X-ray collimator fig. 1a. For non null incidences, the irradiated zone is slightly elongated but it remains close to a disk as shown in fig. $1 \mathrm{~b}$. The specific diffraction conditions are detailled later in the text.

(a)

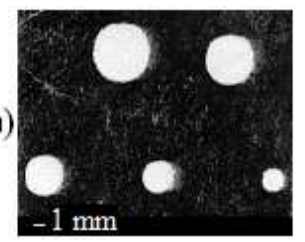

(b)

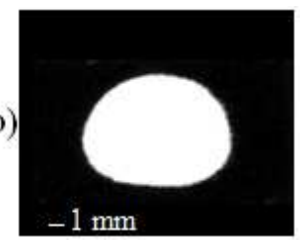

Figure 1. Recording on polaroid film. (a) Various collimations

(b) Shift and deformation of the irradiated zone for $\Psi=29.7^{\circ}$ and $\Psi=-29.7^{\circ}$. 


\section{Stress field convolution and impulse response}

In this section, we propose an experimental procedure to estimate the impulse response of the diffractometer. We call $\sigma_{11}(x, y)$ the local stress at a given point $(x, y)$ on the specimen surface in the stress reference frames $(O, x, y, z)$.We assume that the stress measures $\left\langle\sigma_{11}(x, y)\right\rangle^{*}$ obtained by XRD are the convolution of the local stress field $\sigma_{11}(x, y)$ by the impulse response $h(x, y)$ of the diffractometer: $\left\langle\sigma_{11}(x, y)\right\rangle=\int_{-\infty}^{\infty} \int_{-\infty}^{\infty} h(x-u, y-v) \cdot \sigma_{11}(x, y) d u d v$ plus some Gaussian noise. The most direct way to determine $h(x, y)$ of an X-ray diffractometer would be to perform measurements of a stress field that follows a Dirac distribution $\delta(x, y)$. Since it is pratically impossible to obtain such a stress field, we consider instead a stress field that follows a Heaviside function. Therefore, we consider a EN10084 steel sample containing a grinded and an annealed zones. The annealing treatment relaxes the surface stresses whereas the grinding introduces a residual stress $\sigma_{11}(x, y)$ of approximately $-280 \mathrm{MPa}$ perpendicularly to the grinding direction. The stress $\sigma_{11}(x, y)$ varies brutally from a null value to $-280 \mathrm{MPa}$ with respect to the $(O x)$ direction (cf. fig. 2a). This variation occurs within a range smaller than $100 \mu \mathrm{m}(100 \mu \mathrm{m}$ is the depth at which grinding stresses are usually relaxed [5]). By the specimen design, the stress field does not vary along the $(O y)$ direction. The impulse response studied is only in the $(O x)$ direction and thus it corresponds to the single-dimensional

convolution: $\left\langle\sigma_{11}(x)\right\rangle=\int_{-\infty}^{\infty} h(x-u) \cdot \sigma_{11}(x) d u$

The experimental procedure is as follows. Stress measurements are performed every $100 \mu \mathrm{m}$ along the $(O x)$ axis fig 2a. A diaphragm of $2.6 \mathrm{~mm}$ diameter is used in the collimator and the irradiated disc has an estimated diameter of $4.2 \mathrm{~mm}$. The irradiation time is set to $30 \mathrm{~s}$. Stress measurements reported in fig. $2 \mathrm{~b}$ are obtained by the $\sin ^{2} \psi$ method wich is carried out over $8 \psi$ incidences with opposite pairs of angles. fig. $2 \mathrm{~b}$ clearly shows that the stress goes from 0 to $-280 \mathrm{MPa}$ over a range of $4000 \mu \mathrm{m}$ which is much larger that the zone of zone affected by grinding (c.a. $100 \mu \mathrm{m}$ ). Next, we propose a geometrical model for the spacial convolution of the stress field.
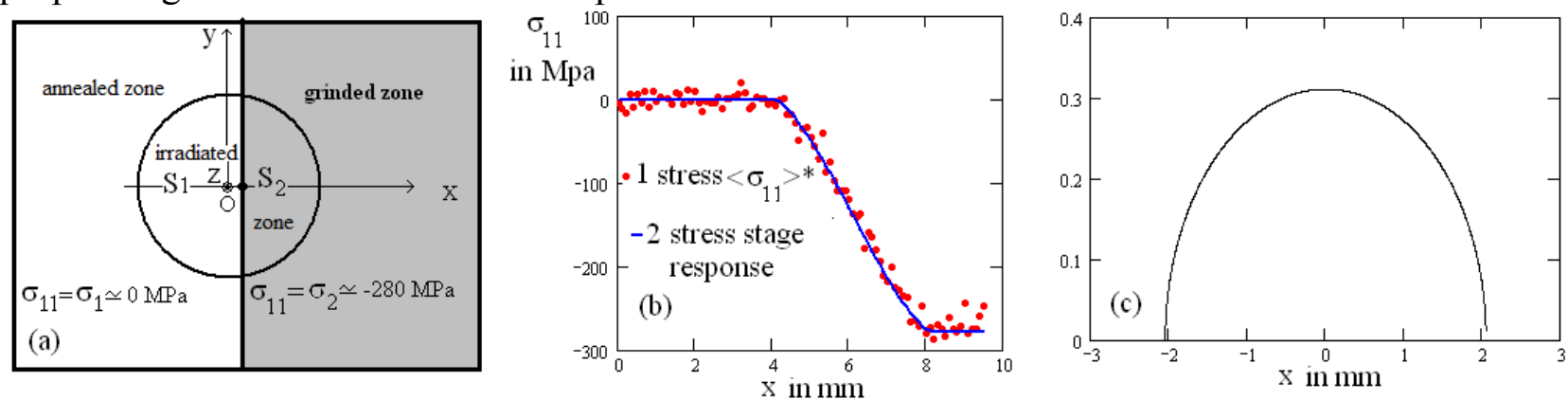

Figure 2. (a) Annealed (left half) and rectified (right half) specimen used in this study.

(b) Response to a stress stage. (c) Impulse response of the diffractometer.

We formulate the following hypothesis.

- The intensity of the incidencial X-ray beam is homogeneous inside the irradiated zone. It has been shown [3] that the intensity distribution is indeed close to a uniform distribution in the case of a collimator diameter larger than $3 \mathrm{~mm}$ and for the same XRD apparatus as the one used in the present investigation.

-The irradiated zone is a disc which shape is weakly affected by the incidence $\psi$ as it will be confirmed in next section.

These two hypothesis allow us to describe the determination of the $\left\langle\sigma_{11}(x)\right\rangle$ stress (fig. 2a) where the convolution window is a disc with an unknow radius $b$ (in connection with the beam size). The stress measures thus corresponds to: 


$$
<\sigma_{11}(x)>=\left(\begin{array}{c}
\sigma_{1}, \text { when } x \leq-b \\
\frac{S_{1} \sigma_{1}+S_{2} \sigma_{2}}{S_{1}+S_{2}}, \text { when }-b \leq x \leq b \\
\sigma_{2}, \text { when } x>b
\end{array}\right)
$$

$S_{1}$ and $S_{2}$, the two parts of irradiated zone fig. 2a, depend on the $x$ position.

This leads to $\pi \frac{<\sigma_{11}(x)>-\sigma_{1}}{\sigma_{2}-\sigma_{1}}=a \cos (u)+u \sqrt{1-u^{2}}$, with: $u=-\frac{x}{b}$ for $-b \leq x \leq b$

For each measure, $\left\langle\sigma_{11}(x)\right\rangle^{*}$ corresponds to a solution $u$ of equation (3). By plotting each value of $u$ versus $x$, we obtain by linear regression a straight line with a slope of $-\frac{1}{b}$. The stress stage response (fig. $2 \mathrm{~b}$ ) is in agreement with the measured $b$ value which is $2.03 \mathrm{~mm}$. We thus obtain a convolution disc with a diameter $2 b$ close to the one of the irradiated (cf. section II, $4.2 \mathrm{~mm}$ )

The derivative of equation (3) gives the impulse response:

$$
h(x)=\left(\begin{array}{c}
2 \sqrt{b^{2}-x^{2}} \text { when }-b \leq x \leq b \\
0 \text { when } x \in R-[-b, b]
\end{array}\right)
$$

The curve of the impulse response is presented fig. 2c. It is observed that with a good approximation, the measured stress is the average value of the stress field in the extent of the irradiated zone.

\section{III) Incidence angles and impulse response symmetry.}

In this section, the influence of the set of $\Psi$ incidences on the stress convolution is evaluated. To this aim, we choose to bend a specimen with an embeded extremity (fig. 3a) leading to a stress field that is spatially varying. A planar stress state is assumed which leads to:

$$
\sigma_{11}(x, y)=\frac{3 F}{2 h^{3} e} y(l-x) \quad \sigma_{12}(x, y)=-\frac{3 F}{4 h^{3} e}\left(h^{2}-y^{2}\right) \text { and } \sigma_{22}=\sigma_{13}=\sigma_{23}=\sigma_{33} \approx 0
$$
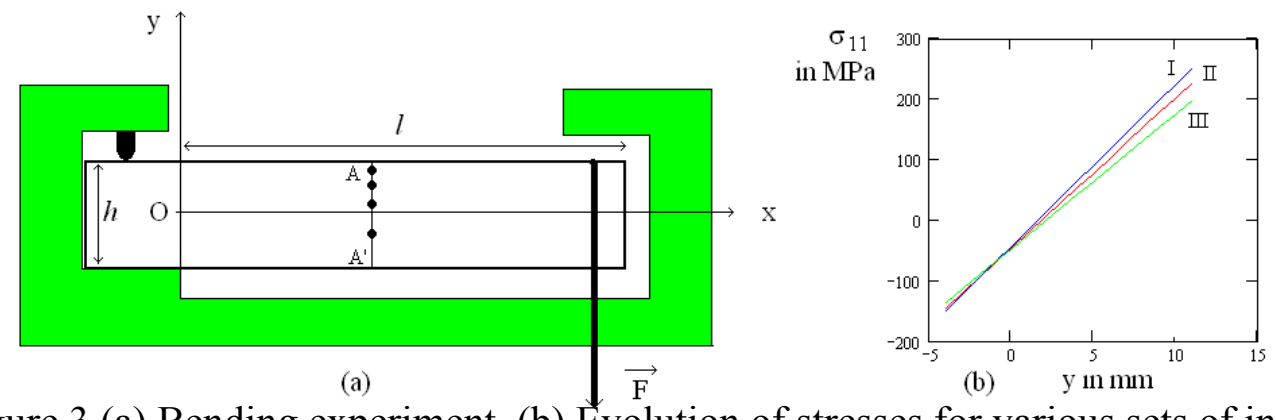

Figure 3.(a) Bending experiment, (b) Evolution of stresses for various sets of incidences.

The diameter of collimator is $2.6 \mathrm{~mm}$. The stress $\sigma_{11}(x, y) *$ is determined at four points along the AA' segment for $y=-4.5 ; 0.5 ; 5.5$ and $10.5 \mathrm{~mm}$ (see fig. 3a). Three different sets I, II and III of incidences are used to analyse the stress. Set I contains more negative incidences, Set III has more positive incidences and Set II include only oposite pairs of incidences (cf. Tab. 1). Result of the stress measurements are given in Tab. 2 and linear regressions on the stress values with respect to the $y$ position are done in fig. 3b. Slightly different evolution of the stress measures (slopes) are found depending on the used incidences. For $y>0$, the stress value is minored for the set III and majored for the set I. Opposite results are obtained for $y<0$. The actual irradiated zone position seems to be shifted when the incidence angle is changed. As the local stress is a function of $x$, the stress measures are found to be modified when the number of positive incidences is not equal to the number of negative incidences. The shift in the stress measures increases with $y$ because the local stress $\sigma_{11}(x, y)$ is also a function $y$. However, the relative difference between the measures of stress I and III with measure II is roughly constant and is only about $10 \%$. 
Table 1 : Set of incidences.

\begin{tabular}{|l|c|c|c|}
\hline Set & I & II & III \\
\hline Set of inci- & $39,2 / 35,8 / 32,3 / 28,6 / 24,5 /$ & $39,2 / 35,8 / 32,3 / 28,6 / 24,5 /$ & $24,5 / 19,8 / 13,8 /-39,2 /$ \\
dences in & $19,8 / 13,8 /-39,2 /-35,8 /-$ & $19,8 / 13,8 /-39,2 /-35,8 /-$ & $-35,8 /-32,3 / 28,6 /$ \\
degres & 32,3 & $32,3 /$ & $-24,5 /-19,8 /-13,8$ \\
& & $-28,6 /-24,5 /-19,8 /-13,8 /$ & \\
\hline
\end{tabular}

Table 2 Stress measurements for the different sets of incidences.

\begin{tabular}{|l|c|c|c|}
\hline Set & I & II & III \\
\hline $\mathrm{y}=-4,5 \mathrm{~mm}$ & -183 & -170 & -149 \\
$\mathrm{y}=0,5 \mathrm{~mm}$ & -8 & -20 & -40 \\
$\mathrm{y}=5,5 \mathrm{~mm}$ & 119 & 100 & 87 \\
$\mathrm{y}=10,5 \mathrm{~mm}$ & 222 & 205 & 180 \\
\hline Slope $(\mathrm{MPa} / \mathrm{mm})$ & 26,84 & 24,9 & 22,28 \\
Intercept point $(\mathrm{MPa})$ & $-230,9$ & $-220,25$ & $-203,3$ \\
\hline
\end{tabular}

\section{Measurement and deconvolution of a spatially-varying stress field}

In this section, we illustrate a deconvolution procedure to obtain the local stress values from experimental stress measurements. Since deconvolution techniques are sensitive to the amount of noise in the experimental data and in order to validate with no ambiguity the proposed deconvolution procedure, we consider an experimental situation where the local stress field is known (e.g. from analytical models). We chose a rectangular specimen containing a trough hole in the middle. We focus on the stress field around the specimen hole when the specimen is deformed under a uniaxial compression.

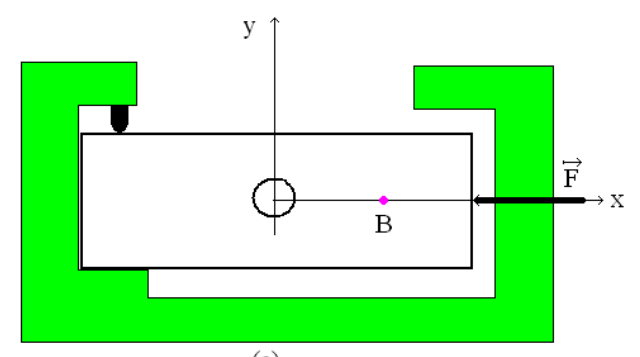

(a)

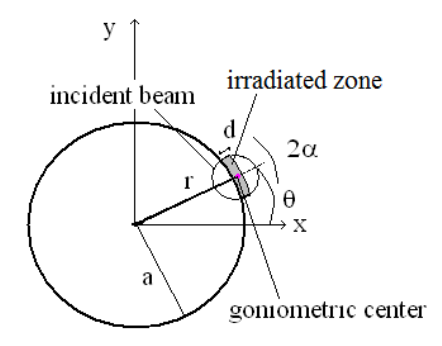

(b)

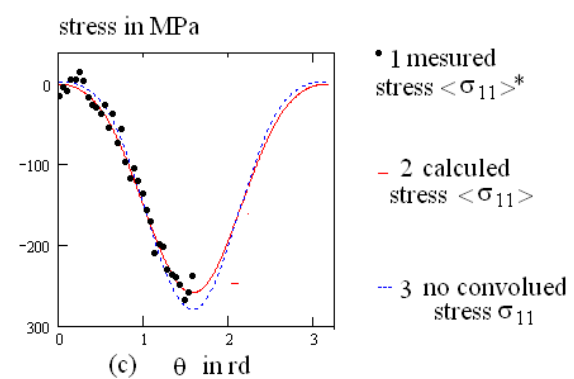

Figure 4 : (a) Compression experiment on a specimen contraining a throuh hole (b)-Irradiated zone

(c)-Stress profile.

The experimental conditions are as follows. In order to remove the influence of the incidence angle upon the irradiation zone geometry, we use a mask containing a hole in the shape of an arc of circle, which acts like a pass-all filter. The irradiated zone is localized in the region $[a-d / 2 ; a+d / 2]$ and $[\theta-$ $\alpha ; \theta+\alpha$ ] (in polar coordinates, fig. $4 \mathrm{~b}$ ) where $a$ is the radius of the specimen hole $a=6 \mathrm{~mm}, d=1 \mathrm{~mm}$ and $\alpha=20^{\circ}$. The mask is fixed, and the compression machine and the specimen are rotating with respect to the center of the specimen hole. Thirty-two stress measurements in the $(O x)$ direction, are performed every $2.8^{\circ}$ in the domain $\theta=[0 ; \pi / 2]$. Incidences are located with respect to the basis II. The irradiation time for each incidence was set to $200 \mathrm{~s}$. An additional stress measurement noted $\sigma$, was performed at point $\mathrm{B}$, in the $(O x)$ direction, far from the hole (along $(O x)$ and in between the specimen hole and the surface where the loading is applied). A value of $\sigma=-112 \mathrm{MPa}$ was obtained. The stress measurements around the specimen hole are reported fig. $4 \mathrm{c} 1$. Next we compare the experimental values with the convoluted stress field calculated from the theoretical stress field. Starting from biharmonic Airy stress functions, the stress components at a point $(r, \theta)$ close to the hole are: 


$$
\begin{gathered}
\sigma_{r r}=\frac{\sigma}{2}\left(1-\frac{a^{2}}{r^{2}}\right)+\frac{\sigma}{2}\left(1+\frac{3 a^{4}}{r^{4}}-\frac{4 a^{2}}{r^{2}}\right) \cos (2 \theta) ; \sigma_{\theta \theta}=\frac{\sigma}{2}\left(1+\frac{a^{2}}{r^{2}}\right)-\frac{\sigma}{2}\left(1+\frac{3 a^{4}}{r^{4}}\right) \cos (2 \theta) ; \\
\sigma_{r \theta}=\frac{\sigma}{2}\left(1-\frac{3 a^{4}}{r^{4}}+\frac{2 a^{2}}{r^{2}}\right) \sin (2 \theta)
\end{gathered}
$$

The $\sigma_{11}(r, \theta)$ stress component is related to the previous stress components by $\sigma_{11}(r, \theta)=\sigma_{t t} \cos ^{2} \theta-\sigma_{r \theta} \sin (2 \theta)+\sigma_{\theta \theta} \sin ^{2} \theta$. As shown previously, the measured stress is the convolution of the local stress over the irradiated surface. Therefore, the convoluted stress over the analyzed surface is: $\left\langle\sigma_{11}(r, \theta)\right\rangle=\frac{1}{2 r d \alpha} \int_{\theta-\alpha}^{\theta+\frac{d}{2}} \int_{\frac{d}{2}}^{r+\frac{d}{2}} \sigma_{11} r d r d \theta$. The theoretical convoluted stress field is shown fig. $4 \mathrm{c} 2$ and a good agreement is found with the experimental stress measurements. It can be noted that the theoretical non-convoluted stress field is slightly different from the convoluted response. The maximum difference of $20 \mathrm{MPa}$ is found at $\theta=\pi / 2$. This value constitutes an estimation of the error made when stress analyses are performed without considering the spatial convolution of the stress measures. Next, we perform a deconvolution procedure of the experimental data $\left\langle\sigma_{11}(r, \theta)\right\rangle^{*}$ in order to obtain the local stress $\sigma_{11}(r, \theta)$. The irradiated zone used to perform the experimental stress measures exhibits a relatively narrow radial dimension $(d=1 \mathrm{~mm})$. Therefore, it is reasonable to consider that the convolution is only carried over $\theta \in[-\alpha ;+\alpha]$. The convolution equation becomes: $\left\langle\sigma_{11}(\theta)\right\rangle=\sigma_{11}(\theta) * h(\theta)$ where $h(\theta)=1 / 2 \alpha$ for a rectangular convolution window of size $[-\alpha ;+\alpha] . h(\theta)=0$ everywhere else. Considering the expected axial symmetry of the stress field under investigation, we use the method based on the Fourrier transform of the convolution equation. By virtue of the convolution theorem, the deconvoluted stress field is: $\sigma_{11}(\theta)=F T^{-1}\left[\frac{F T\left(<\sigma_{11}(\theta)>\right)}{F T(h(\theta))}\right]$ where FT is a Fourrier transform. The deconvolution can be simple in practice but the deconvolution procedure is an ill-posed inverse problem and noise in the experimental data can lead to instability in the solution. Various regularization techniques have been proposed in the literature [6-9] to find stable solutions. Here, we use the truncation of the Fourrier transform method where the Fourrier transform of the experimental data points $F T\left(<\sigma_{11}(\theta)>^{*}\right)$ are set to zero for frequencies larger that a cut-off frequency $\omega_{c}$. Beyond this frequency, fluctuations in the Fourrier transform are due to the experimental noise. fig. 5a presents the real and imaginary parts of the non-truncated Fourrier transform of $\left\langle\sigma_{11}(\theta)>*\right.$ where most of the useful signal is seen in the low frequencies domain. In practice, the truncation is performed via the multiplication of $\left\langle\sigma_{11}(\theta)\right\rangle$ by a function $f(\omega)=1$ for $\omega \leq \omega_{c}$ and $f(\omega)=\exp \left[c\left(\omega_{c}-\omega\right)\right]$ elsewhere. Here we consider the parameters $c$ and $\omega_{c}$ as fitting parameters and we will show that they can be used to discuss the stability of the corresponding solution. To discuss the relative stability of the deconvoluated signal solution, $c$ is varied by steps $\Delta c=1$ (in Fourier space) in the range [1,7] and $\omega_{c}$ is varied by steps $\Delta \omega_{c}=1$ in the range $[1,20]$. For every couple $\left(c, \omega_{c}\right)$, the curve solution $\sigma_{11}(\theta)$ is compared to the height curve solutions $\sigma_{11}(\theta)$ obtained for height the neighboring $\left(c^{n}=\left\{\begin{array}{c}c-\Delta c \\ c \\ c+\Delta c\end{array}\right\} ; \omega_{c}^{n}=\left\{\begin{array}{c}\omega_{c}-\Delta \omega_{c} \\ \omega_{c} \\ \omega_{c}+\Delta \omega_{c}\end{array}\right\}\right)$ couples. We introduce a relative stability criterion defined as the average of the quadratic differences between a solution $\sigma_{11}(\theta)$ obtained for the couple $\left(c, \omega_{c}\right)$ and the height neighboring $\sigma_{11}(\theta)$ curves. fig. $5 \mathrm{~b}$ displays the mapping of the stability criterion as a function of $\left(c, \omega_{c}\right)$. Stable solutions are coded in blue and 
instable solutions are in red. The solution shape $\sigma_{11}(\theta)$ is also given for few $\left(c, \omega_{c}\right)$ domains and compared with the theoretical local stress at the center of the irradiated zone fig. $5 \mathrm{c}$. A large domain where the solution is stable can be clearly seen and a good agreement is obtained with the theoretical local stress field.
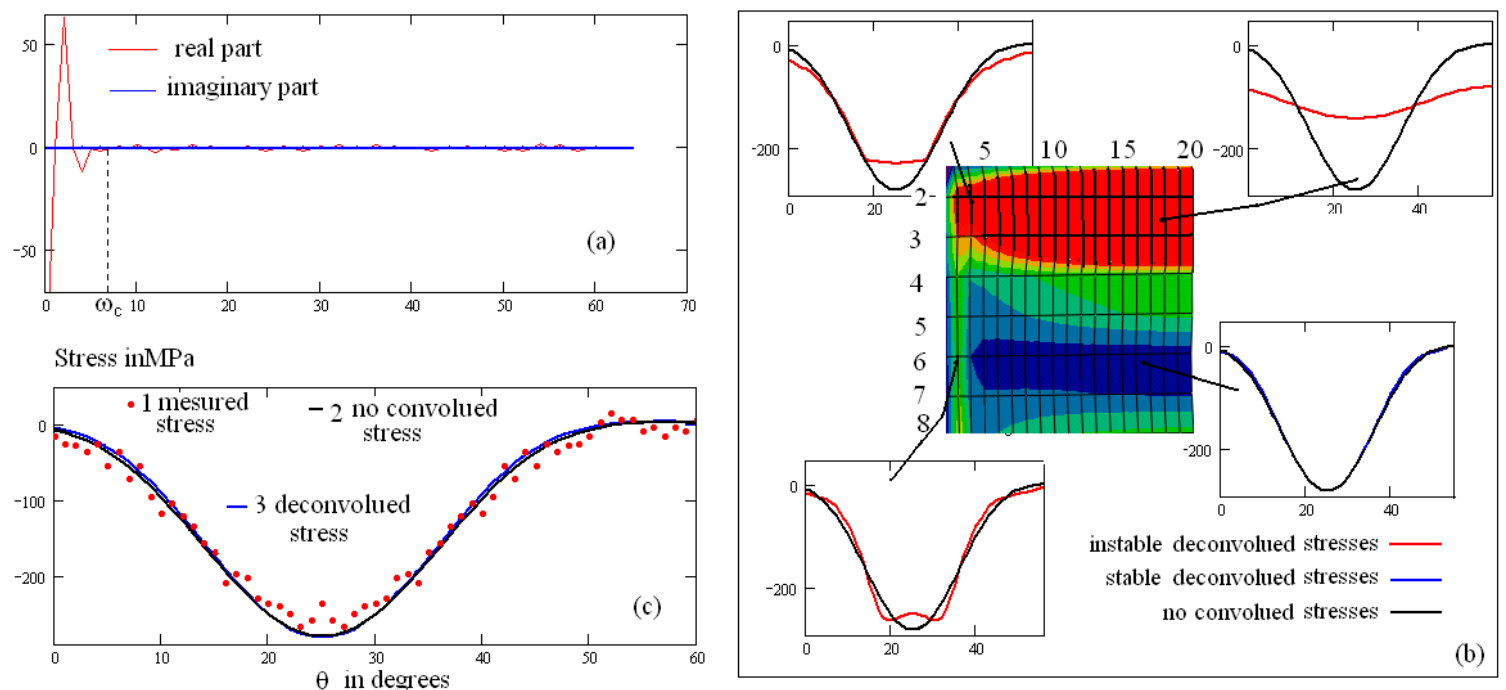

Figure 5 (a) Fourrier transform of the experimental stress measures (b) Mapping of the solution stability (c) Stable solution compared with the expected local stress.

\section{Conclusion}

Stress measurements using XRD result from the spatial convolution of the local stress over the irradiated surface of the specimen. For the experimental setup used here, we show that this convolution is simply the average of the local stress field over the analysed surface and the impusional response of the diffractometer is directly in connection with the circular shape of the irradiated surface. The irradiated surface is found to become slightly assymmetrical with respect to the goniometric center when incidence angles are varied. We show that the local stress field can be obtained from the experimental measurements using a deconvolution procedure based on the Fourier transform of the convolution equation. A stable solution is found when a rationalization procedure is performed and when noise in the experimental data is limited.

\section{References}

[1] Norme XP A 09-285. Méthode d'essai pour l'analyse des contraintes résiduelles par diffraction des rayons X. mai 1999

[2] C. Kahloun, C. Badawi (1994). X-ray Analysis. The case of substantial stress gradients and strong heterogeneity, Proceeding of ICRS4, Balitmore 1994, p252.

[3] V. Hennion, J.M. Sprauel and H. Michaud. Contribution to residual-stress evaluation in highstress-gradient zones by X-ray diffraction. Journal of applied crystallography (2000) .

[4] M Desvignes. L. Castex. J.M. Sprauel. Nouvelle appareil de diffractométrie X pour analyse des contraintes et dosage d'austénite. Colloque Siemens Grenoble 23 au 26 avril 1985.

[5] Mémoires Techniques du CETIM n³3. 1978 p38-39

[6] A.N. Tikonov. V Arsenine Méthode de résolution des problèmes mal posés. Moscou 1976

[7] L.B. Lucy. An iterrative technic for the rectification of observed images. Astronomical Journal, 754-754, 1974

[8] A. Tarantola. Inverse problem theory-methode for data fitting and model parameter estimation. Elsevier 1987

[9] Van Cittert, P.H.,Z.Phys. 69 (1931) 298. 